\title{
AFib amyloidosis
}

INSERM

\section{Source}

INSERM. (1999). Orphanet: an online rare disease and orphan drug data base. AFib amyloidosis. ORPHA:93562

AFib amyloidosis is a rare, hereditary amyloidosis with primary renal involvement characterized by fibrinogen A-alpha-chain amyloid deposition predominantly in the kidney glomeruli and clinically presenting with hypertension, uremia, nephrotic syndrome slowly progressing to end-stage renal disease. Extra-renal involvement is possible, due to neurological, cardiac, visceral and vascular amyloid deposition. 